\title{
Oral dydrogesterone in frozen-thawed embryo transfer cycles
}

\author{
Luma Caroline Gomes Mattos de Macedo ${ }^{1 *}$ (D), Mario Cavagna Neto ${ }^{2 \dagger}$, Artur Dzik² (D), Andressa do \\ Rosário Rocha² (D), Sônia Maria Rolim Rosa Lima ${ }^{1}$
}

\section{SUMMARY}

OBJECTIVE: The aim of this study was to compare the use of micronized vaginal progesterone and oral dydrogesterone in the endometrial preparation for frozen-thawed embryo transfer.

METHODS: This was a randomized, controlled, open, two-armed clinical trial, with women undergoing frozen-thawed embryo transfer along with hormone replacement therapy for endometrial preparation, between September 2019 and February 2021. A total of 73 patients were randomly selected and orally administered $40 \mathrm{mg} /$ day dydrogesterone (dydrogesterone group, $\mathrm{n}=36$ ) or $800 \mathrm{mg}$ /day micronized vaginal progesterone (micronized vaginal progesterone group, $n=37$ ), after endometrial preparation with transdermal estradiol. The main outcome was a viable ongoing pregnancy with 12 weeks of gestation as evaluated by ultrasound.

RESULTS: The reproductive outcomes in frozen-thawed embryo transfer cycles were similar, with pregnancy rates in the dydrogesterone and micronized vaginal progesterone treatment groups being, respectively, 33.3 and $32.4 \%$ at 12 weeks pregnancy (confidence interval=-22.420.6, $\mathrm{p}=0.196$ ).

CONCLUSIONS: The use of oral dydrogesterone may be a more patient-friendly approach to endometrial preparation in frozen-thawed embryo transfer cycles, avoiding undesirable side effects and discomfort resulting from vaginal administration, while also providing similar reproductive results. KEYWORDS: Dydrogesterone. Embryo transfer. Progesterone. Fertilization in vitro. Infertility.

\section{INTRODUCTION}

More efficient cryopreservation strategies, such as the development of the vitrification technique and positive results pertaining to their safety, have progressively increased the use of frozen-thawed embryo transfer (FET) over the past decade ${ }^{1}$. Using a protocol with an antagonist and triggering the final follicular maturation with an agonist, followed by a "freezeall" strategy and embryo transfer in a subsequent cycle, is an effective option for preventing the ovarian hyperstimulation syndrome $(\mathrm{OHS})$ and leads to high rates of live births ${ }^{2,3}$. Other advantages and the applicability of FET are multiple pregnancy risk prevention by the elective transfer of one or a few fresh embryos, thereby allowing for the cryopreservation surpluses and carrying out a preimplantation genetic study $y^{4}$.

Several different protocols are available for endometrial preparation. Still, there is no consensus on the most effective procedure for preparing the endometrium before FET in normo-ovulatory patients ${ }^{1}$. The methods of endometrial preparation for FET can be divided into natural and medicated (artificial) cycles. During the natural cycle, participants were only monitored, without receiving any pharmacological intervention before ovulation. In contrast, in the medicated cycle, estrogen was administered to achieve endometrial proliferation and suppression of follicular growth, and progesterone is administered to mimic the luteal phases.

Progesterone can be administered via oral, vaginal, rectal, subcutaneous, or intramuscular routes. All these forms of administration appear to have similar efficacy ${ }^{6}$. However, the vaginal route is the standard of treatment at most in vitro fertilization (IVF) centers. One explanation for this is that the vaginal route does not involve the first hepatic passage and also provides higher and sustained serum concentrations than does the oral route. Nevertheless, all forms of progesterone administration can have side effects, such as discharge and bleeding by the vaginal route; and daily intramuscular administration can lead to pain at the injection site ${ }^{6}$.

In view of the side effects and difficulty involved in parenteral and vaginal administration, the oral route would be an option for luteal phase support and to prepare endometrium for the transfer of thawed embryos. Since micronized progesterone does not have good intestinal absorption, dydrogesterone (DYD) appears to be a better option. It is a retro-steroid with good oral bioavailability and high selectivity for progesterone receptors. It can be used at lower oral doses to mimic

\footnotetext{
${ }^{1}$ Irmandade da Santa Casa de Misericórdia de São Paulo, School of Medical Sciences, Department of Obstetrics and Gynecology - São Paulo (SP), Brazil. ${ }^{2}$ Centro de Referência da Saúde da Mulher, Hospital Pérola Byington, Human Reproduction - São Paulo (SP), Brazil.

*Corresponding author: luma_karol@hotmail.com

†In Memoriam.
}

Conflicts of interest: the authors declare there is no conflicts of interest. Funding: none.

Received on September 08, 2021. Accepted on September 08, 2021. 
the luteal phase due to its better bioavailability and the progestogenic activity of its metabolites ${ }^{7}$. The use of this medication is considered safe during pregnancy ${ }^{8}$.

Data from prospective studies on the support for the luteal phase in IVF with fresh embryo transfer have shown that oral DYD is as effective as micronized vaginal progesterone (MVP), with better patient satisfaction rates ${ }^{9,10}$. Safety results were similar between both groups ${ }^{11}$.

However, the vast majority of studies have been carried out with IVF cycles using fresh embryo transfer, whereas those using frozen embryos remain scarce. For this reason, we were interested in comparing MVP and oral DYD in the endometrial preparation for the transfer of frozen-thawed embryos.

\section{METHODS}

This was a randomized, controlled, parallel, open clinical trial, with two groups of women undergoing FET along with hormone replacement therapy for endometrial preparation, at the Assisted Reproduction Service at Hospital Pérola Byington, in partnership with the Faculty of Medical Sciences at Santa Casa de São Paulo, conducted between September 2019 and February 2021.

Patients' characteristics such as age, body mass index (BMI), as calculated by the formula weight $/ h_{\text {height }}^{2}\left(\mathrm{~kg} / \mathrm{m}^{2}\right)$ and categorized based on the criteria defined by World Health Organization, referral for assisted reproduction techniques, endometrial thickness after 10-12 days of estrogen use, number of embryos transferred, embryonic stage and quality, biochemical pregnancy (positive $\beta$-human chorionic gonadotropin hormone [HCG] test), clinical pregnancy (visualization of the fetal heartbeat by ultrasound at six weeks of gestational age), and pregnancy at 12 weeks of gestation were routinely input into the electronic medical records in our database.

The patients were randomly divided into two groups: one group used oral DYD, whereas the other used MVP (Figure 1).

\section{Inclusion criteria}

Women undergoing embryo cryopreservation and FET due to the risk of OHS, surplus embryos following failed pregnancy after the fresh transfer, the absence of transfer due to an inappropriate endometrium, or patients who underwent preimplantation genetic diagnosis were included in this study.

\section{Exclusion criteria}

Women with an endometrium smaller than $7 \mathrm{~mm}$ after endometrial preparation with estrogen, history of recurrent miscarriages (history of $\geq 3$ spontaneous miscarriages), severe male factor, uterine diseases, and the presence of hydrosalpinx and those who had a dominant follicle even after estrogen administration were excluded from the study.

\section{Endometrial preparation protocol}

Estradiol administration was initiated transdermally (Oestrogel ${ }^{\circledR}$ Besins Healthcare, Belgium) at a $6 \mathrm{mg} /$ day dose on the second day of the menstrual cycle. After 10-12 days of estrogen therapy, a blood sample was collected, and a transvaginal ultrasound was performed to assess the estradiol, luteinizing hormone (LH), progesterone levels, and endometrial thickness. If the thickness of the endometrium was $<7 \mathrm{~mm}$, estrogen therapy was extended for another five days, and the dose increased to $8 \mathrm{mg} /$ day. When a $7-\mathrm{mm}$ thick, triple-line endometrium was observed, accompanied by serum progesterone concentrations $<1.5 \mathrm{ng} / \mathrm{mL}$, we started administering MVP $800 \mathrm{mg} /$ day (Utrogestan ${ }^{\circledR} 200 \mathrm{mg}$, Besins Healthcare) in one of the groups; in the other group, $40 \mathrm{mg} /$ day (Duphaston ${ }^{\circledR}$ $10 \mathrm{mg}$; Abbott BV, Netherlands) DYD was given orally while maintaining estradiol administration. Embryo transfer was performed after progesterone administration on day three for day three embryos and on day five for blastocysts.

Supplementation with estrogen and progestogen was continued at the same dose until the pregnancy test, performed 15 days after embryo transfer. This support was continued up to 10-12 weeks in viable pregnancies. Ultrasonography was performed during the sixth, eighth, and twelfth weeks of amenorrhea.

\section{Embryo transfer}

Embryos were obtained from fertilization in vitro cycles or intracytoplasmic sperm injection, vitrified, and heated on day three or at the blastocyst stage. All embryo transfers were performed with ultrasound guidance. The number of embryos transferred, their stage, and their quality were recorded. If at least one good-quality embryo was transferred, quality was classified as Q+. The criteria for $\mathrm{Q}+$ quality was the same as those for day three embryos: 6-10 cells with less than 20\% fragmentation according to the Holte classification ${ }^{12}$; whereas for blastocysts, expanded to hatched blastocysts with internal cell mass and trophectoderm A or B quality (from 4B upward) according to the Garden classification ${ }^{13}$.

\section{Sample size calculation and statistical analysis}

For calculating the sample size, the comparison of proportions was considered; the reference values were those from LOTUS $\mathrm{I}^{10}$. A difference of 0.281 was adopted, with a significance level of $5 \%$, and a test power of $80 \%$. In this case, we found $\mathrm{n}=36$ per group. 
For the bivariate analysis, a proportion comparison was performed using the normal approximation method with a significance level $=0.05 ; 95 \%$ confidence intervals (CIs) were constructed for proportion differences.

The computer software used for conducting the statistical analysis was Statistical Package for the Social Sciences (SPSS) version 13.0 for Windows.

\section{RESULTS}

Initially, a total of 111 cycles of patients who were to undergo FET with hormone replacement therapy for endometrial preparation was randomized into one of the treatment groups. In general, 65.8\% (73/111) reached the end of the study after exclusion criteria were applied, 37 of whom were in the MVP group and 36 in the DYD group.

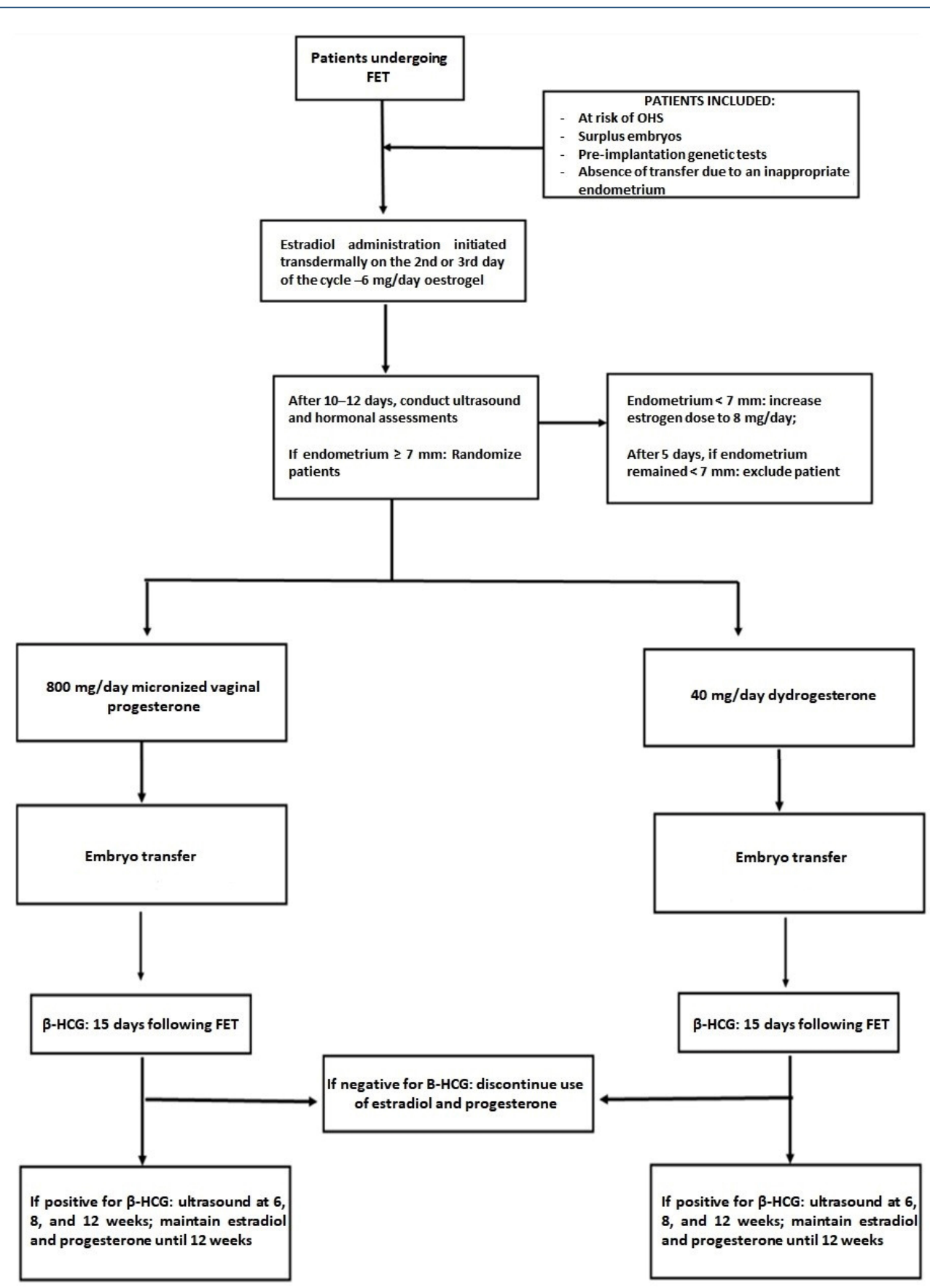

FET: frozen-thawed embryo transfer; OHS: ovarian hyperstimulation syndrome; $\beta$-HCG: human chorionic gonadotropin hormone; US: ultrasound.

Figure 1. Research protocol flowchart. 
The patients' characteristics were similar between the two treatment groups and are summarized in Table 1. The patients' age ranged from $23-40$ years, with a mean of 33.2 years $( \pm 4.4)$ : in the DYD group, it was $34.1( \pm 4.4)$ years, and in the MVP group, it was $32.3( \pm 4.3)$ years.

Most patients did not have comorbidities. In relation to BMI, $64.4 \%$ of them had a BMI lower than $30 \mathrm{~kg} / \mathrm{m}^{2}$, with an average of $25.8 \mathrm{~kg} / \mathrm{m}^{2}$ (overweight). Hypothyroidism, with appropriate treatment, was described in $8.1 \%(n=3)$ of the individuals in the MVP group versus $11.4 \%(n=4)$ in the DYD group.

The number of embryos transferred was also similar between the two treatment groups. There was a greater number of embryos that were transferred in the blastocyst stage as compared to D3.
In most cycles (73.6\%), at least one good-quality (Q+) embryo was transferred; this was similar in the two groups $(77.1 \%$ in the VP group and $70.3 \%$ in the DYD group).

The reproductive outcomes in FET cycles were similar to the two progesterone supplementation methods (oral DYD vs. vaginal progesterone), as demonstrated by the pregnancy rate at 12 weeks of gestation (Table 2). Pregnancy rates in the DYD and MVP treatment groups were respectively: biochemistry 38.9 and $37.8 \%(\mathrm{p}=0.189,95 \% \mathrm{CI}-23.4-21.2)$, clinical 33.3 and $35.1 \%(\mathrm{p}=0.192,95 \% \mathrm{CI}-20.0-23.6)$, and 12 weeks pregnancy 33.3 and $32.4 \%(p=0.196,95 \% C I-22.4-20.6)$. The rate of pregnancy loss in the first trimester was similar in the groups, with two cases having been observed in each group.

Table 1. Patients' characteristics and treatment results.

\begin{tabular}{|c|c|c|c|}
\hline & Oral DYD (36) & MVP (37) & Total $(n=73)$ \\
\hline Mean age, years (SD) & $34.1(4.4)$ & $32.3(4.3)$ & $33.2(4.4)$ \\
\hline \multicolumn{4}{|l|}{ Age, $n(\%)$} \\
\hline$<35$ years & $17(43.5)$ & $22(56.4)$ & $39(53.4)$ \\
\hline$\geq 35$ years & $19(55.8)$ & $15(44.1)$ & $34(46.6)$ \\
\hline Mean BMI, kg/m² (SD) & $25.2(5.0)$ & $26.5(5.7)$ & $25.8(5.3)$ \\
\hline $\begin{array}{l}\text { Mean endometrial thickness on the day progesterone was } \\
\text { administered }(\mathrm{mm})\end{array}$ & $9.0(1.7)$ & $9.2(1.6)$ & $9.1(1.7)$ \\
\hline \multicolumn{4}{|l|}{ Embryonic stage, n (\%) } \\
\hline D3 & $14(38.9)$ & $16(43.2)$ & $30(41.1)$ \\
\hline Blastocyst & $22(61.1)$ & $21(56.8)$ & $43(58.9)$ \\
\hline \multicolumn{4}{|l|}{ Number of embryos transferred, n (\%) } \\
\hline 1 & $17(47.2)$ & $16(43.2)$ & $33(45.2)$ \\
\hline 2 & $16(44.4)$ & $19(51.4)$ & 35 (47.9) \\
\hline$>2$ & $3(8.3)$ & $2(5.4)$ & $5(6.8)$ \\
\hline \multicolumn{4}{|l|}{ Embryonic quality, n (\%) } \\
\hline$Q^{+}$ & $27(77.1)$ & $26(70.3)$ & $53(73.6)$ \\
\hline$Q_{-}^{-}$ & 8 (22.9) & $11(29.7)$ & $19(26.4)$ \\
\hline
\end{tabular}

DYD: dydrogesterone; MVP: micronized vaginal progesterone; SD: standard deviation; BMI: body mass index.

Table 2. Pregnancy rates after treatment in the two study groups.

\begin{tabular}{|c|c|c|c|c|}
\hline \multirow{2}{*}{ Pregnancy } & \multicolumn{2}{|c|}{$\%(n / N)$} & \multirow{2}{*}{$\begin{array}{l}\text { Difference inpregnancy rate } \\
\text { (Oral DYD - MVP) }\end{array}$} & \multirow{2}{*}{$95 \% \mathrm{Cl}$} \\
\hline & Oral DYD & MVP & & \\
\hline \multicolumn{5}{|l|}{ Pregnancy rate } \\
\hline Biochemical pregnancy, n (\%) & $38.9(14 / 36)$ & $37.8(14 / 37)$ & 1.1 & $-23.4-21.2$ \\
\hline Clinical pregnancy, n (\%) & $33.3(12 / 36)$ & $35.1(13 / 37)$ & 1.8 & $-20.0-23.6$ \\
\hline Pregnancy at 12 weeks, n (\%) & $33.3(12 / 36)$ & $32.4(12 / 37)$ & 0.9 & $-22.4-20.6$ \\
\hline
\end{tabular}

Clinical pregnancy: six weeks of gestational age; DYD: dydrogesterone; MVP: micronized vaginal progesterone; $\mathrm{Cl}$ : confidence interval. Biochemical pregnancy: positive $\beta$-human chorionic gonadotropin hormone test two weeks after embryo transfer. 


\section{DISCUSSION}

This study demonstrated that the reproductive outcomes in FET cycles were similar to the two methods of progesterone supplementation (oral DYD versus vaginal progesterone) with regard to the study's primary objective, which is the rate of ongoing pregnancies, and its secondary objectives, which are the biochemical and clinical pregnancy rates. Accordingly, we can provide supporting evidence for the use of oral DYD in FET, similar to the results already established in the literature for fresh embryo transfer.

Several studies have shown oral DYD as an alternative to MVP to support the luteal phase in IVF cycles when using fresh embryo transfer ${ }^{6,9,11,14-16}$. Among these, the randomized, double-blind, multicenter phase III clinical trial (LOTUS I) for luteal phase support has notably demonstrated that oral DYD is as effective as MVP, as determined by pregnancy rates at 12 weeks of gestation ${ }^{10}$.

It is important to highlight the relevance of our results since several randomized clinical trials are showing that the oral DYD is an alternative to MVP to support the luteal phase when using fresh embryo transfer. Nevertheless, there is a lack of studies comparing the efficacy of these two types of progestogens in FET cycles, which would probably be the most effective way to assess the two types of treatment, since the corpus luteum would not secrete progesterone in these cases.

Our findings are also supported by ample evidence from a meta-analysis comparing oral DYD with MVP for supporting the luteal phase in women undergoing IVF with the transfer of fresh and/or frozen-thawed embryos, showing similar reproductive outcomes with the two progestogens ${ }^{17}$. However, this study did not take into account the clinical heterogeneity that may exist due to the main endocrinological differences between both IVF protocols in cycles with either fresh embryo transfer or FET ${ }^{11,18}$. To reduce this bias, in our study, we only evaluated FET cycles and excluded patients who had a dominant follicle after estrogen administration.

Our findings with FET cycles are corroborated by the results described by Rashidi et al. ${ }^{19}$, who conducted a randomized, controlled, single-blind study with 180 women undergoing FET. They were recruited and allocated into three groups (i.e., Group A was given $50 \mathrm{mg}$ of intramuscular progesterone

\section{REFERENCES}

1. Mackens S, Santos-Ribeiro S, van de Vijver A, Racca A, Van Landuyt L, Tournaye H, et al. Frozen embryo transfer: a review on the optimal endometrial preparation and timing. Hum Reprod. 2017;32(11):2234-42. https://doi.org/10.1093/humrep/dex285

2. Blockeel C, Drakopoulos P, Santos-Ribeiro S, Polyzos NP, Tournaye H. A fresh look at the freeze-all protocol: a SWOT analysis. Hum Reprod. 2016;31(3):491-7.https://doi.org/10.1093/humrep/dev339 twice daily; Group B: $20 \mathrm{mg}$ oral DYD twice daily; Group C: $400 \mathrm{mg}$ MVP twice daily). Their results showed that oral DYD is as effective as intramuscular and vaginal progesterone. However ${ }^{19}$, they did not evaluate either ovulation or luteinization that can occur in $5 \%$ of cycles, which is one of the limitations considered by the authors.

We observed similar rates of ongoing pregnancies in the two research groups, and no patient discontinued treatment due to side effects or intolerance to the progestogens used. The $40 \mathrm{mg}$ DYD dose (the highest dose safely used in other studies) was chosen based on data disclosed in the literature and on recommendations from IVF specialists ${ }^{11,19}$.

Nonetheless, this study has some limitations. The analysis of the results was originally performed to consider the rate of ongoing pregnancies, but the rate of live births may be of greater clinical interest. The findings of this research are strengthened by the selection of an appropriate sample size comprising 73 randomized individuals, the fact that both treatment arms are well balanced, and the use of broad eligibility criteria. Yet, with a larger sample size, we could have obtained more robust evidence. Therefore, there is a need for further work comparing the effectiveness of these two types of progestogens in FET cycles in a larger group.

\section{CONCLUSIONS}

The use of oral DYD may be a more patient-friendly approach to endometrial preparation in FET cycles, avoiding undesirable side effects and discomfort resulting from vaginal administration, while providing similar reproductive results.

\section{AUTHORS' CONTRIBUTIONS}

LCGMM: Conceptualization, Data curation, Formal Analysis, Writing - original draft, Writing - review \& editing. MCN: Conceptualization, Data curation, Formal Analysis, Writing original draft, Writing - review \& editing. AD: Conceptualization, Data curation, Formal Analysis, Writing - original draft, Writing - review \& editing. ARR: Data curation, Formal Analysis. SMRRL: Conceptualization, Data curation, Formal Analysis, Writing - original draft, Writing - review \& editing.

3. Zhu Q, Chen Q, Wang L, Lu X, Lyu Q, Wang Y, Kuang Y. Live birth rates in the first complete IVF cycle among 20687 women using a freeze-all strategy. Hum Reprod. 2018;33(5):924-9. https://doi. org/10.1093/humrep/dey044

4. Pandian Z, Bhattacharya S, Ozturk O, Serour G, Templeton A. Number of embryos for transfer following in-vitro fertilisation or intra-cytoplasmic sperm injection. Cochrane Database Syst Rev. 2009;(2):CD003416. https://doi.org/10.1002/14651858. CD003416.pub3 
5. Paulson RJ. Hormonal induction of endometrial receptivity. Fertil Steril. 2011;96(3):530-5. https://doi.org/10.1016/j. fertnstert.2011.07.1097

6. van der Linden M, Buckingham K, Farquhar C, Kremer JA, Metwally M. Luteal phase support for assisted reproduction cycles. Cochrane Database Syst Rev. 2015;2015(7):CD009154. https://doi.org/10.1002/14651858.CD009154.pub3

7. Schindler AE. Progestational effects of dydrogesterone in vitro, in vivo and on the human endometrium. Maturitas. 2009;65(Suppl 1):S3-11. https://doi.org/10.1016/j.maturitas.2009.10.011

8. Queisser-LuftA. Dydrogesterone use during pregnancy: overview of birth defects reported since 1977. Early Hum Dev. 2009;85(6):375-7. https://doi.org/10.1016/j.earlhumdev.2008.12.016

9. Barbosa MW, Silva LR, Navarro PA, Ferriani RA, NastriCO, MartinsWP. Dydrogesterone vs progesterone for luteal-phase support: systematic review and meta-analysis of randomized controlled trials. Ultrasound ObstetGynecol.2016;48(2):161-70.https://doi.org/10.1002/uog.15814

10. Tournaye H, Sukhikh GT, Kahler E, Griesinger G. A Phase III randomized controlled trial comparing the efficacy, safety and tolerability of oral dydrogesterone versus micronized vaginal progesterone for luteal support in in vitro fertilization. Hum Reprod. 2017;32(10):2152. https://doi.org/10.1093/humrep/dex266

11. Griesinger G,Blockeel C, Kahler E,Pexman-FiethC,Olofsson JI,Driessen $S$, et al. Dydrogesterone as an oral alternative to vaginal progesterone for IVF luteal phase support: a systematic review and individual participant data meta-analysis. PLoS One. 2020;15(11):e0241044. https://doi.org/10.1371/journal.pone.0241044

12. Holte J, Berglund L, Milton K, Garello C, Gennarelli G, Revelli A, et al. Construction of an evidence-based integrated morphology cleavage embryo score for implantation potential of embryos scored and transferred on day 2 after oocyte retrieval. Hum Reprod. 2007;22(2):548-57. https://doi.org/10.1093/humrep/del403
13. Gardner DK, Schoolcraft WB. Culture and transfer of human blastocysts. Curr Opin Obstet Gynecol. 1999;11(3):307-11. https://doi.org/10.1097/00001703-199906000-00013

14. Griesinger G, Blockeel C, Sukhikh GT, Patki A, Dhorepatil B, Yang DZ, et al. Oral dydrogesterone versus intravaginal micronized progesterone gel for luteal phase support in IVF: a randomized clinical trial. Hum Reprod. 2018;33(12):2212-21. https://doi. org/10.1093/humrep/dey306

15. Griesinger G, Blockeel C, Tournaye H. Oral dydrogesterone for luteal phase support in fresh in vitro fertilization cycles: a new standard? Fertil Steril. 2018;109(5):756-62. https://doi.org/10.1016/j. fertnstert.2018.03.034

16. Saharkhiz N, Zamaniyan M, Salehpour S, Zadehmodarres S, Hoseini $\mathrm{S}$, Cheraghi L, et al. A comparative study of dydrogesterone and micronized progesterone for luteal phase support during in vitro fertilization (IVF) cycles. Gynecol Endocrinol. 2016;32(3):213-7. https://doi.org/10.3109/09513590.2015.1110136

17. Barbosa MWP, Valadares NPB, Barbosa ACP, Amaral AS, Iglesias JR, Nastri CO, et al. Oral dydrogesterone vs. vaginal progesterone capsules for luteal-phase support in women undergoing embryo transfer: a systematic review and meta-analysis. JBRA Assist Reprod. 2018;22(2):148-56. https://doi.org/10.5935/15180557.20180018

18. Ghobara T, Gelbaya TA, Ayeleke RO. Cycle regimens for frozen-thawed embryo transfer. Cochrane Database Syst Rev. 2017;7(7):CD003414. https://doi.org/10.1002/14651858. CD003414.pub3

19. Rashidi BH, Ghazizadeh M, Nejad EST, Bagheri M, Gorginzadeh M. Oral dydrogesterone for luteal support in frozen-thawed embryo transfer artificial cycles: A pilot randomized controlled trial. Asian Pac J Reprod. 2016;5:490-4. https://doi.org/10.1016/j. apjr.2016.10.002 